С.С. КАРДАШЕВА, К.М.Н., Ю.О. ШУЛЬПЕКОВА, К.М.Н., В.И. ЛЕЩЕНКО, К.М.Н.

Первый Московский государственный медицинский университет им. И.М. Сеченова

\title{
ГАСТРОЭЗОФАГЕАЛЬНЫЙ РЕФЛЮКС:
}

\section{НОВЫЕ ПЕРСПЕКТИВЫ ЛЕЧЕНИЯ}

\begin{abstract}
В статье представлены данные эпидемиологических исследований по распространенности симптомов гастроэзофагеальной рефлюксной болезни (ГЭРБ), факторы риска и проявления ГЭРБ. Особое внимание уделено индивидуальному терапевтическому подходу и выбору современного эффективного средства для лечения - ИПП декслансопразола.
\end{abstract}

Ключевые слова: гастроэзофагеальная рефлюксная болезнь, неэрозивная рефлюксная болезнь, эрозивный эзофагит, терапия, ИПП, декслансопразол.

\section{S.S. KARDASHEVA, PhD in medicine, Y.O. SHULPEKOVA, PhD in medicine, V.I. LESCHENKO, PhD in medicine Sechenov First Moscow State Medical University GASTROESOPHAGEAL REFLUX: NEW THERAPEUTIC PROSPECTS}

The article provides the data of epidemiologic studies on frequency of symptoms of the gastroesophageal reflux disease (GERD), risk factors and GERD manifestations. Special emphasis is made on the individual therapeutic approach and choice of the modern effective drug for therapy - PPI Dexlansoprazol.

Keywords: gastroesophageal reflux disease, non-erosive reflux disease, erosive esophagitis, therapy, proton pump inhibitor (PPI), Dexlansoprazol.

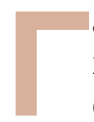

астроэзофагеальная рефлюксная болезнь хроническое рецидивирующее заболевание, обусловленное нарушением моторно-эвакуаторной функции органов гастроэзофагеальной зоны и характеризующееся регулярно повторяющимся рефлюксом в пищевод желудочного и/или дуоденального содержимого, что приводит к повреждению дистального отдела пищевода с развитием в нем функциональных нарушений и/или дистрофических изменений неороговевающего многослойного плоского эпителия, катарального, эрозивного или язвенного рефлюкс-эзофагита, сопровождается характерными симптомами, а у части больных - цилиндроклеточной метаплазией (пищеводом Барретта) [1].

По данным отечественных эпидемиологических исследований, распространенность рефлюксных симптомов в российской популяции составляет 40-60\%; критерии ГЭРБ определяются у 20-25\%. В мире сохраняется тенденция к росту распространенности этого заболевания. Один из наиболее весомых факторов риска развития ГЭРБ - наличие висцерального ожирения - типичного заболевания сегодняшнего дня. Таким образом, ГЭРБ во многих случаях можно рассматривать как одно из «гастроэнтерологических проявлений» системной патологии метаболического синдрома [2, 3]. В свою очередь, ГЭРБ может сочетаться с нарушениями сна и, по-видимому, способствовать поддержанию повышенного артериального давления.

Типичные симптомы ГЭРБ - изжога и регургитация, возникающие регулярно (раз в неделю и более) и ухудшающие качество жизни. Внепищеводные проявления с установленной патогенетической связью с гастроэзофагеальным рефлюксом - кашель, бронхиальная астма, ларингит, эрозии эмали зубов.

Гастроэзофагеальная рефлюксная болезнь в 60\% случаев протекает в форме неэрозивной рефлюксной болез- ни (НЭРБ), другая форма заболевания - эрозивный эзофагит развивается реже. Пищевод Барретта относится к осложнениям и, по различным данным, выявляется у 6-12\% пациентов с характерными симптомами ГЭРБ. Однако реальная распространенность пищевода Барретта в популяции значительно выше. Риск развития рака при пищеводе Барретта составляет в среднем 0,5\% в год.

Проявления ГЭРБ существенно снижают качество жизни больных [4]. Четкая корреляция тяжести симптомов и степени нарушения целостности слизистой пищевода не обнаруживается. Если в патогенезе при НЭРБ важную роль играют нарушения моторики со снижением клиренса пищевода и сенситизации слизистой, то при формах, сопровождающихся нарушением целостности эпителия, развитии эрозивно-язвенных изменений, пищевода Барретта и аденокрациномы, по-видимому, основная роль принадлежит более грубым, трудноустранимым, механическим и анатомическим факторам, способствующим поддержанию контакта слизистой с рефлюктатом. Так, при эрозивном рефлюкс-эзофагите чаще наблюдаются тяжелая степень недостаточности нижнего пищеводного сфинктера (по классификации Hill), аксиальные грыжи пищеводного отверстия диафрагмы, выше частота и продолжительность патологического рефлюкса с pH $<4$ [5]. Лица с эрозивным эзофагитом чаще страдают морбидным ожирением; есть данные о влиянии употребления алкогольных напитков (в особенности крепких) и курения на предрасположенность к эрозивному эзофагиту и пищеводу Барретта [6]. Возможно, в основе рецидивирующего эрозивного процесса также играет роль наследственно обусловленное снижение экспрессии белка межклеточных контактов Е-кадхерина, что способствует проявлению повреждающего действия рефлюктата. В патогенезе ГЭРБ изучается роль изменений микрофлоры верхних отделов желудочно-кишечного тракта, в частности преобладания грамнегативных 
микроорганизмов, способствующих повышению продукции провоспалительных цитокинов и снижению пищеводного клиренса.

Диагностика, тактика лечения и наблюдения пациентов с различными формами ГЭРБ с современных позиций подробно изложена в национальных руководствах, в частности в Клинических рекомендациях Российской гастроэнтерологической ассоциации от 2014 г. [1].

\section{ОБЩИЕ ПРИНЦИПЫ ТЕРАПИИ}

Индивидуальный подход при назначении лечения основывается на оценке частоты и интенсивности симптомов, наличия и степени тяжести эзофагита, анамнеза предшествующих обострений заболевания. Во многих случаях решающее значение имеет изменение стиля жизни, коррекция массы тела, отказ от курения и злоупотребления алкоголем. Для лечения пациента с НЭРБ и умеренной эпизодической изжогой (два раза в неделю или реже) и отсутствием эрозивного эзофагита в анамнезе можно применить принцип «step-up-терапии» - терапии нарастающей интенсивности. Начать можно с рекомендаций изменения образа жизни, диеты и приема антагонистов $\mathrm{H}_{2}$-рецепторов гистамина (H2RA) в низкой дозе (половина от стандартной два раза в день) с добавлением антацидов (табл.) [8]. Если эффект от лечения в течение 2 нед. недостаточный, необходимо увеличить дозу H2RA до стандартной и продолжить лечение как минимум 2 нед. При сохранении симптомов рекомендуется отменить H2RA и назначить ингибиторы протонной помпы сначала в низкой дозе, с возможным увеличением до стандартной [8]. Продолжительность лечения после достижения контроля симптомов должна составить не менее 8 нед. Систематический анализ рандомизирован-

\section{Таблица. Стандартные дозы антисекреторных препаратов при ГЭРБ}

\begin{tabular}{|c|c|}
\hline Препарат & Стандартная дозировка при ГЭРБ \\
\hline \multicolumn{2}{|c|}{ Блокаторы $\mathrm{H}_{2}$-рецепторов гистамина } \\
\hline циметидин & 400 мг × 2 р/день \\
\hline ранитидин & 150 мг × 2 р/день \\
\hline фамотидин & 20 мг × 2 р/день \\
\hline низатидин & 150 мг × 2 р/день \\
\hline \multicolumn{2}{|c|}{ Ингибиторы протонной помпы } \\
\hline омепразол & 40 мг 1 р/день \\
\hline лансопразол & 30 мг 1 р/день \\
\hline пантопразол & 40 мг 1 р/день \\
\hline рабепразол & 20 мг 1 р/день \\
\hline эзомепразол & 40 мг 1 р/день \\
\hline декслансопразол & 30 мг или 60 мг 1 р/день \\
\hline
\end{tabular}

ных исследований, изучавших влияние изменения образа жизни и диеты на симптомы ГЭРБ и данные внутрипище-

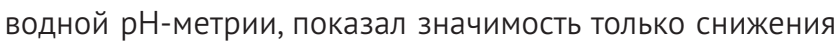
избыточного веса и подъема головного конца кровати.

\section{Диагностика, тактика лечения и наблюдения пачиентов с различными формами ГЭРБ с современных позиций подробно изложена в национальных руководствах, в частности в Клинических рекомендациях Российской гастроэнтерологической ассочиации от 2014 г.}

Последнее показано при ночных симптомах, внепищеводных легочных и ларингеальных симптомах. Диетические рекомендации включают избирательное ограничение пищевых триггеров гастроэзофагеального рефлюкса (жирная, острая пища, кофе, шоколад), если пациент отмечает корреляцию своих симптомов с приемом определенной пищи. Применение антацидов и алгинатов при ГЭРБ ограничивается приемом «по требованию» для купирования редкой изжоги и назначением совместно с антисекреторными препаратами в первые дни лечения для быстрого купирования изжоги. Средняя продолжительность действия H2RA составляет 4-10 ч, эти препараты более эффективно, чем антациды, купируют симптомы, однако их эффективность в заживлении эрозивного эзофагита недостаточна. Применение H2RA для поддерживающей терапии ограничивается развитием тахифилаксии спустя 2-6 нед. со значительным снижением эффективности.

Эрозивный эзофагит, частые (более 2 раз в неделю) и интенсивные симптомы, существенно снижающие качество жизни пациента, служат показанием к первоначальному назначению ингибиторов протонной помпы (ИПП) в стандартной дозе для максимально быстрого облегчения симптомов («step-down-терапия») (табл.) [1, 8]. ИПП максимально эффективны при приеме утром, за 30 минут перед первым приемом пищи, поскольку плотность Н-К-АТФазы на париетальной клетке коррелирует с продолжительностью тощакового периода (ночь). Все ИПП, независимо от дозы, превосходят по эффективности контроля симптомов и заживления эрозивного эзофагита H2RA: в среднем в 86\% случаев после 8 нед. терапии удается достичь клинико-эндоскопической ремиссии. По данным метаанализа рандомизированных клинических исследований, статистически достоверных различий в эффективности различных ИПП не установлено. Через 4 нед. лечения эрозии в пищеводе заживали у 49-91\% пациентов, через 8 нед. - у 71-99\% пациентов. Постоянная поддерживающая терапия ИПП изначально рекомендуется при тяжелом эзофагите (С- и D-степени) и при пищеводе Барретта [1]. Пациентам с НЭРБ и эрозивным эзофагитом А-В-степени рекомендуется попытаться отменить антисекреторную терапию: через 8 нед. при хорошем контроле симптомов - переход на низкую дозу ИПП и затем замена ИПП на H2RA c последующей отменой [1, 8]. 
Приблизительно 65\% пациентов с НЭРБ и большинство с эрозивным эзофагитом отмечают рецидив симптомов после отмены антисекреторной терапии. В случае раннего рецидива симптомов в течение 3 месяцев после отмены лечения рекомендуется проведение контрольной ЭГДС и рассмотрение варианта постоянной поддерживающей терапии ИПП в минимальной дозе [10]. В метаанализе показано, что режим приема ИПП «по требованию» в качестве поддерживающей терапии менее эффективен, чем постоянная поддерживающая терапия, как в плане клинической, так и эндоскопической ремиссии, хотя и более удобен для пациентов [11].

В настоящее время всем больным пищеводом Барретта рекомендуется постоянная антисекреторная терапия ИПП и эндоскопическое наблюдение, эндоскопическая абляция показана при выявлении дисплазии метаплазированного эпителия. На фоне антисекреторной терапии возможна частичная регрессия метаплазии эпителия.

При планировании длительной терапии ИПП пациентам, инфицированным Helicobacter pylori, целесообразно проведение эрадикационной терапии [12].

\section{ЛЕЧЕНИЕ ГЭРБ ПРИ БЕРЕМЕННОСТИ И ЛАКТАЦИИ}

Первоначальный подход к лечению ГЭРБ во время беременности включает рекомендации изменения образа жизни, соблюдения диеты и приема антацидов, алгинатов или сукральфата [13]. Не рекомендуются антациды, содержащие бикарбонат натрия и трисульфат магния. При недостаточном эффекте рекомендуется назначить H2RA, больше данных накоплено по применению ранитидина во время беременности. Исследований, изучавших применение ИПП во время беременности, меньше, чем по применению H2RA, однако все публикации свидетельствуют о безопасности ИПП [13]. Больше данных накоплено по применению омепразола, лансопразола и пантопразола во время беременности. Метаанализ 7 наблюдательных исследований не выявил увеличения риска врожденных дефектов, спонтанных абортов или преждевременных родов [13].

\section{ФАРМАКОЛОГИЯ ИПП}

После приема внутрь ИПП всасываются в тонкой кишке. Действующее вещество накапливается в зонах с наиболее низкими значениями $\mathrm{pH}$ и в области секреторных канальцев париетальных клеток, где $\mathrm{pH}=1 \div 2$, концентрация ИПП почти в 1000 раз превышает таковую в крови. В этих условиях происходит протонирование ИПП с образованием активной формы - сульфенамида [13, 14]. Последний необратимо связывается с цистеиновым остатком $\mathrm{H}^{+} / \mathrm{K}^{+}$-АТФазы (протонной помпы) и блокирует ее. Кислотопродукция восстанавливается по мере встраивания вновь синтезированных молекул $\mathrm{H}^{+} / \mathrm{K}^{+}$-АТФазы в мембрану париетальных клеток. Скорость активации пантопразола практически прекращается при $\mathrm{pH}=4$. Скорость образования сульфенамида из омепразола, эзомепразо- ла и лансопразола снижается в 2 раза при $\mathrm{pH}=4$, рабепразола - при $\mathrm{pH}=4,9[14,15]$.

ИПП метаболизируются в микросомах печени с участием субъединиц цитохрома Р450 - СРР2С9, СРР2С19, CYP2D6 и CYP3A4; при этом наблюдается конкуренция с другими субстратами [8, 9]. Наибольшее значение имеет взаимодействие ИПП с СҮР2C19 и СYР3А4. По выраженности угнетения функции СҮР2С19 за лансопразолом следуют омепразол, эзомепразол, рабепразол и пантопразол; по силе влияния на CYP3A4 за пантопразолом следуют омепразол, эзомепразол, рабепразол, лансопразол $[1,2,7]$. Ген СҮР2С19 отличается полиморфизмом, что влияет на выраженность терапевтического эффекта ИПП. CYP2C19 участвует в метаболизме многих лекарств, поэтому большое практическое значение придают влиянию ИПП именно на эту субъединицу. Один из важнейших аспектов применения ИПП - вероятность снижения эффекта антитромбоцитарного препарата клопидогрела за счет конкуренции за взаимодействие с СYP2C19 и снижения скорости образования активных метаболитов клопидогрела $[9,10]$. CYP3А4 также играет важную роль в метаболизме лекарств; ее активность существенно варьирует. CYP3A4 экспрессируется также на апикальной мембране кишечного эпителия, что может влиять на биодоступность лекарств и «эффект первого прохождения через печень».

\section{Преобладание определенного стереоизомера может влиять на длительность поддержания терапевтической концентрации в плазме; эта особенность учитывалась при создании декслансопразола - правовращающего изомера лансопразола}

Фармакокинетика ИПП может зависеть от соотношения содержания лево(S)- и право(R или D)-вращающих стереоизомеров действующей молекулы. Так, создание эзомепразола - левовращающего изомера омепразола способствовало уменьшению различий в биодоступности и действия препарата у «быстрых» и «медленных» метаболизаторов. Для других ИПП создание «чистого» препарата на основе лишь одного изомера не всегда дает такое разительное преимущество в нивелировании различий скорости метаболизма у «быстрых» и «медленных» метаболизаторов. Однако преобладание определенного стереоизомера может влиять на длительность поддержания терапевтической концентрации в плазме; эта особенность учитывалась при создании декслансопразола правовращающего изомера лансопразола [11].

\section{НОВЫЕ ПЕРСПЕКТИВЫ ЛЕЧЕНИЯ ЭРОЗИВНОГО ЭЗОФАГИТА}

Лечение эрозивных рефлюксных поражений пищевода может представлять особенную сложность вследствие высокой частоты рецидива: на стадиях эзофагита А и В, по лос-анджелесской классификации, она составляет поряд- 
ка 15-23\%, на стадиях C и D - 24-41\% в течение 6 месяцев от начала терапии [12]. Столь высокую частоту рецидива объясняют наличием трудноустранимых факторов, поддерживающих патологический рефлюкс. Поэтому многие эксперты обосновывают целесообразность проведения противорецидивной терапии ИПП в течение полугода.

В качестве одних из наиболее эффективных средств в лечении эрозивного рефлюкс-эзофагита в течение последних лет рассматривались рабепразол и эзомепразол, что определялось способностью этих препаратов обеспечивать максимальное время с рН < 4 в пищеводе, необходимое для заживления дефектов слизистой, а также удобством приема (во многих случаях - 1 р/день) [18-20]. С 2014 г. в России в арсенал самых эффективных средств лечения ГЭРБ и эрозивного эзофагита вошел препарат Дексилант ${ }^{\circledR}$ (декслансопразол) производства компании Takeda [33]. Декслансопразол представляет собой правовращающий (R- или D-) стереоизомер лансопразола (тогда как лансопразол - смесь R- и S-изомеров). R-изомер взаимодействует с протонной помпой с той же степенью родства, что и S, однако его кинетика характеризуется площадью под фармакокинетической кривой (AUC), в 3-5 раз превышающей таковую для S-изомера, поскольку характеризуется более медленным печеночным метаболизмом [22].

Дексилант ${ }^{\circledR}$ выпускается в виде капсул с модифицированным высвобождением. Действующее вещество содержится в капсулах в виде гранул двух типов, защищенных оболочкой, которая растворяется при различных значениях рН. Таким образом, препарат характеризуется уникальным двухэтапным высвобождением: первый этап быстрое высвобождение 25\% дозы происходит в начальном отделе двенадцатиперстной кишки при рН 5,5 через 1-2 ч после приема препарата; второй этап - отсроченное высвобождение $75 \%$ дозы в дистальном отделе тонкой кишки при рН 6,8 примерно через 4-5 ч. [23]. Абсорбция при приеме внутрь составляет $\geqslant 76 \%$. Таким образом, формируется «двухпиковый» график концентрации препарата в плазме, способствующий более длительной циркуляции действующего вещества, что, в частности, доказано в фармакокинетическом исследовании, где проводилось сравнение с лансопразолом [12]. Двухэтапное высвобождение позволяет заблокировать вновь синтезированные и «регенерированные» после первоначального связывания молекулы протонной помпы. Такая динамика высвобождения позволяет преодолеть ограничения, свойственные фармакодинамике и фармакокинетике «традиционных» ИПП; такие ограничения, в частности, связаны с возникновением ночного кислотного прорыва и необходимостью приема препаратов до еды. Таким образом, Дексилант ${ }^{\circledR}$ обладает выраженным и наиболее оптимальным по времени действием на кислотопродукцию в сравнении с другими ИПП. Это единственный ИПП, поддерживающий оптимальный уровень $\mathrm{pH}>4$ в течение 17 часов и позволяющий эффективно контролировать симптомы рефлюксной болезни у большинства больных в течение суток [9, 20, 24]. Вследствие длитель- ной циркуляции AUC декслансопразола существенно повышается, хотя значение максимальной концентрации препарата в плазме $\left(C_{\max }\right)$ значительно не нарастает.

Дексилант ${ }^{\circledR}$ зарегистрирован для лечения ГЭРБ (как НЭРБ, так и эрозивного эзофагита), а также в качестве поддерживающей терапии после лечения эрозивного эзофагита. Препарат выпускается в дозах 30 и 60 мг, предназначенных для приема 1 р/сут.

\section{Дексилант ${ }^{\circledR}$ обладает выраженным и наиболее оптимальным по времени действием на кислотопродукцию в сравнении с другими ИПП. Это единственный ИПП, поддерживающий оптимальный уровень $\mathrm{pH}>4$ в течение 17 часов и позволяющий эффективно контролировать симптомы рефлюксной болезни у большинства больных в течение суток}

Выраженный эффект декслансопразола показан в сравнительных исследованиях. В перекрестном исследовании с однократным приемом препарата в дозе 60 мг и эзомепразола 40 мг и проведением 24-часовой внутрижелудочной $\mathrm{pH}$-метрии показано, что достигнутые значения $\mathrm{pH}$, в особенности во второй половине дня, были выше после приема декслансопразола [14].

Дексилант ${ }^{\circledR}$ можно принимать независимо от приема пищи и времени суток (в отличие от «традиционных» ИПП, которые рекомендуется принимать за 30 мин до завтрака). Это подтверждают результаты перекрестного исследования с участием здоровых добровольцев: не обнаружено существенных различий $\mathrm{C}_{\max }$ и показателей 24-часовой внутрижелудочной рН-метрии, если препарат принимался натощак, за 30 или 5 минут до еды либо через 30 минут после еды [15]. Также не отмечено значимых различий во времени приема препарата в течение дня [16].

В двух двойных слепых плацебо-контролируемых исследованиях показана высокая эффективность декслансопразола в лечении эрозивного эзофагита. В одном приняли участие 4092 пациента, которые были рандомизированы в группы лечения лансопразолом 30 мг, декслансопразолом 60 мг, декслансопразолом 90 мг в день. Частота заживления эрозий в группе получавших декслансопразол 60 мг составила 92-93\%, 90 мг - 93-95\%, среди получавших лансопразол - 86-92\%. Таким образом, эффективность декслансопразолом оказалась выше (хотя различия не были статистически достоверными) [17]. В другой работе назначение декслансопразола в дозе 60 мг оказалось более эффективным в заживлении эрозий у пациентов с морбидным ожирением (индексом массы тела >30 мг/м²) [18].

Применение декслансопразола эффективно в профилактике рецидивов эрозивного эзофагита. На период 6-месячного наблюдения пациенты с зажившими эрозиями были рандомизированы в группу плацебо, а также в группу лечения декслансопразолом в дозах 30 или 60 мг. В группе плацебо ремиссия сохранялась у 27,2\% больных, 
среди получавших декслансопразол 30 мг - у 74,9\% и среди получавших 60 мг - у 82,5\%. И хотя различия в целом оказались недостоверными, при выделении пациентов с тяжелым эзофагитом - стадий C и D - дозировка 60 мг показала статистически значимое преимущество над 30 мг (85\% vs 63\%) [19].

Показано, что лечение декслансопразолом повышает качество жизни пациентов с ГЭРБ, в том числе улучшает качество сна, продуктивность на работе, повышает повседневную активность. Повышение качества жизни, достигнутое на фоне терапии «традиционными» ИПП, может стать еще более отчетливым при переводе на лечение декслансопразолом [12].

\section{Применение препарата Дексилант ${ }^{\circledR}$ обеспечивает эффективный контроль над изжогой на протяжении суток, надежное заживление эрозий и язв (в 92-93\% случаях) к 8-й нед. терапии и поддержание стойкой ремиссии эрозивного эзофагита}

Таким образом, применение препарата Дексилант ${ }^{\circledR}$ обеспечивает эффективный контроль над изжогой на протяжении суток, надежное заживление эрозий и язв (в 92-93\% случаях) к 8-й нед. терапии и поддержание стойкой ремиссии эрозивного эзофагита. Для заживления эрозий препарат рекомендуется применять в дозе 60 мг 1 р/день до 8 нед., в качестве поддерживающей терапии - 30 мг один раз в день до 6 месяцев, для лечения НЭРБ - 30 мг один раз в день в течение 4 нед. Отмена декслансопразола не приводит к нарастанию симптоматики и не сопровождается усилением гипергастринемии, что повышает комплаенс пациентов [12].

\section{ПРОБЛЕМА НЕДОСТАТОЧНОЙ ЭФФЕКТИВНОСТИ ТЕРАПИИ ИПП}

10-15\%, а по некоторым данным, до 40\% пациентов с ГЭРБ, особенно с тяжелым эзофагитом, отмечают недостаточную эффективность стандартных доз ИПП [12]. Среди возможных причин следует обратить внимание на недостаточную приверженность к лечению и несоблюдение правильного режима приема препарата (например, перед завтраком). Таким образом, вопрос о приверженности к лечению и режиму приема препарата - это первое, с чего необходимо начать. Стоит отметить, что препарат Дексилант ${ }^{\circledR}$ способствует поддержанию выполнения назначенных рекомендаций благодаря комфортным условиям приема - один раз в день, не привязывая прием препарата к режиму питания. Но, согласно популяционным исследованиям, остается проблема несвоевременного прекращения приема ИПП при облегчении симптоматики или эпизодического приема.

Если при должном соблюдении режима лечения ответа на терапию ИПП в стандартной дозе в течение 4 нед. не отмечено, ситуацию можно оценивать как «рефрактерную ГЭРБ». Часть случаев объясняется инди- видуальными особенностями метаболизма препаратов, а именно полиморфизмом гена СРР2C19, играющего наиболее важную роль в Азии, в меньшей степени в Европе. Продолжительность действия ИПП у «быстрых метаболизаторов» сокращается, что приводит к недостаточному эффекту и «ночным кислотным прорывам». В части случаев ( $\approx 20 \%)$ эффективна смена препарата на другой, в частности на препарат более пролонгированного действия, либо удвоение дозы - добавление вечернего приема ИПП (или H2RA) перед ужином. ГЭРБ, рефрактерная к «традиционным» ИПП, - важная ниша для применения декслансопразола в силу пролонгированного действия этого препарата и удобства приема (1 p/день), что уменьшает влияние фактора некомплаентности [12]. Добавление прокинетиков к терапии ИПП на современном этапе не имеет доказанного клинического значения.

При рефрактерности также следует критически пересмотреть правильность постановки диагноза «ГЭРБ». Со сходными симптомами могут протекать другие заболевания пищевода и желудка - лекарственный, эозинофильный эзофагит, ахалазия, гастопарез, синдром руминации, аэрофагия, красный плоский лишай, синдром Золлингера - Эллисона. В таких случаях необходим соответствующий пересмотр терапии.

Недавно опубликованы обнадеживающие результаты рандомизированного двойного слепого исследования ИПП нового типа вонопразана («калий-конкурентного ИПП») в лечении эрозивного эзофагита, резистентного к терапии «традиционными» ИПП [20].

\section{ПРОБЛЕМА НЕЖЕЛАТЕЛЬНЫХ ЯВЛЕНИЙ ПРИ ПРИЕМЕ ИПП}

Подавление продукции соляной кислоты теоретически может повлечь за собой нарушение всасывания определенных пищевых компонентов и наклонность $\mathrm{k}$ кишечным инфекциям, дополнительное изменение фармакокинетики лекарств [21]. К середине 2000-х гг. уже накопилось достаточное количество публикаций для подведения промежуточных итогов и метаанализов с оценкой нежелательных явлений.

В отношении абсорбции железа, которая зависит от секреции соляной кислоты и содержания витамина С в желудочном соке, однозначных данных не получено. Считается, что ИПП могут снижать биодоступность витамина С, однако при наблюдении в течение 7 лет пациентов, ежедневно принимавших ИПП, клинически значимых нарушений не установлено [22, 23]. В случае мальабсорбции можно назначать препараты железа.

Столь же неопределенной, недоказанной можно считать и ассоциацию приема ИПП с риском мальабсорбции кальция и развития переломов костей. Вероятнее всего, после приема пищи уровень секреции не столь низкий, чтобы существенно повлиять на абсорбцию кальция. В двух исследованиях высокого качества такие побочные эффекты не показаны $[24,25]$, тогда как в других работах определенная связь прослеживалась. 


\section{ИННОВАЦИОННАЯ ТЕХНОЛОГИЯ ДВОЙНОГО ВЫСВОБОЖДЕНИЯ- ДЛИТЕЛЬНЫЙ КОНТРОЛЬ СИМПТОМОВ ГЭРБ ${ }^{1-4}$}
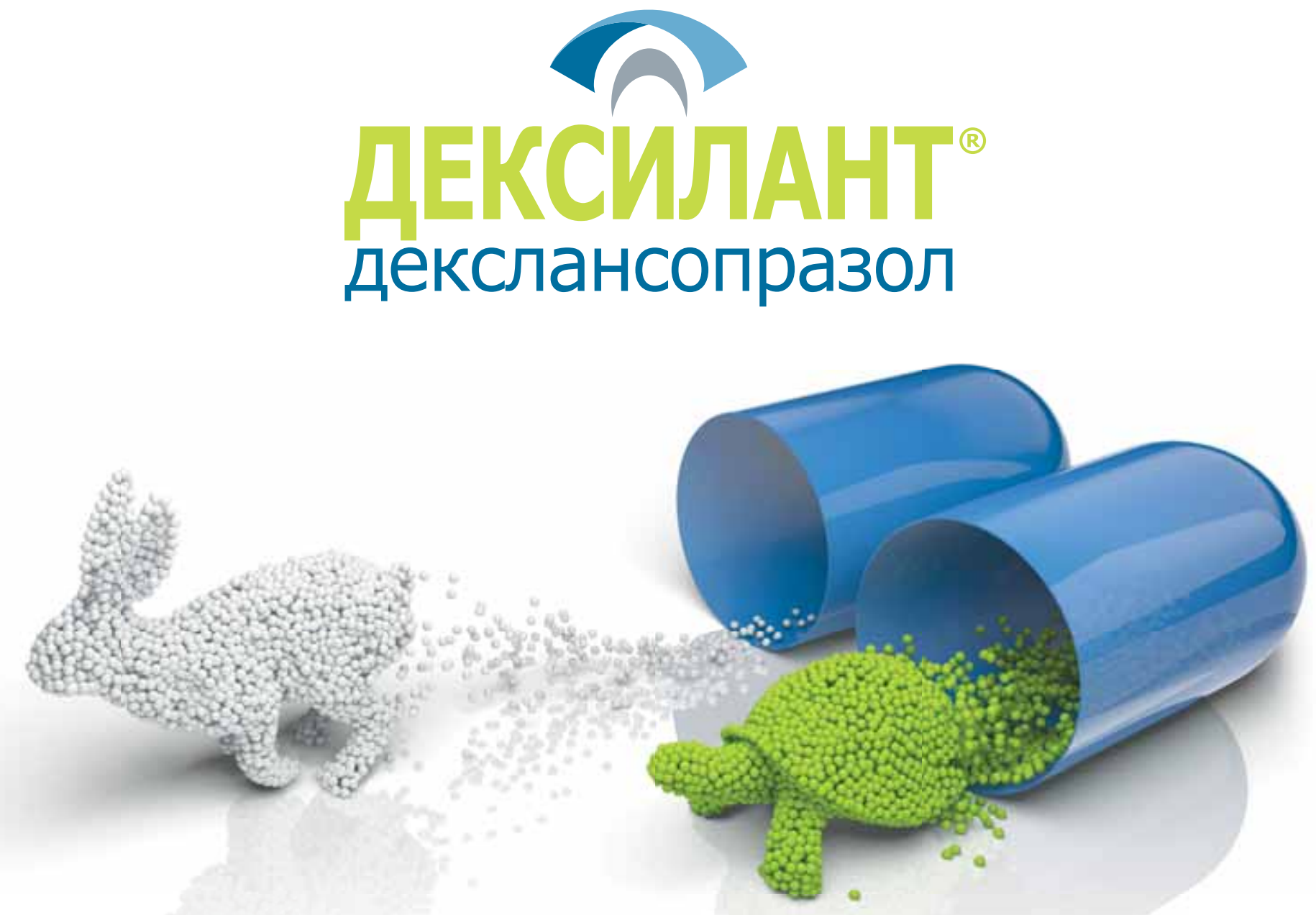

- Контроль симптомов до 24 часов

- Одна капсула в сутки ${ }^{2}$

- Не зависит от приёма пищи란

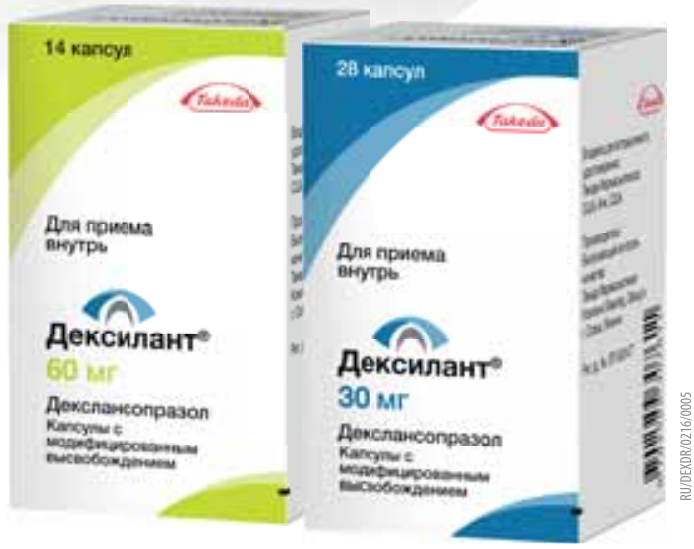

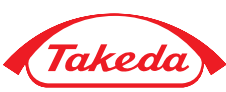

000 «Такеда Фармасьютикалс" 119048 , г. Москва ул. Усачева, дом 2, стр. 1 Тел.: +7 (495) 9335511 ; факс: +7 (495) 5021625 www.takeda.com.ru
Информация для специалистов здравоохранения. Подробнее о применении и противопоказаниях читайте в инструкции. 1. Vakily Met al. Curr Med Res Opin 2009;25:627-38;

3. Homepa nateнtros: 6,664,276-15 December 2020; 6,939,971 - 15 December 2020;

Торговое название: Дексилант". Активное действующее вещество: декслансопразол. Лекарственная форма и дозировка: капсулы с модифицированным высвобождением 30 мг, 60 мг. Показания к применению:

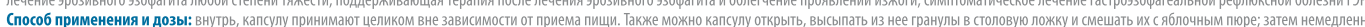
не разжевывая, проглотить. Леченше эрозивного ззофагита любой степени тяжести. Рекомендованной дозой является 60 мг 1 раз в сутки. Курс лечения - 8 недель. Поддерживающая терапия после лечения эрозивного эзофагита

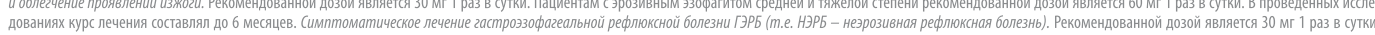
Курс лечения - 4 недели. Полная информация по способу применения и дозам представлена в инструкции по медицинскому применению. Противопоказания к применению: повышенная чувствительность клюбому из компонентов препарата, совместное применение сингибиторами протеаз ВиЧ (атазанавир, нелфинавир), возраст до 18 лет, беременность, период лактации. Препарат содержит сахарозу, поэтому его применение не рекомендовано пациентам снаследственной непереносимостью фруктозы, глюкозо-галактозной мальабсорбцией или сахаразно-изомальтазной недостаточностью. Побочное действие: наиболее частыми нежелательными побочными указания: перед началом лечения декслансопразолом следует исключить возможность злокачественного новообразования, поскольку препарат может маскировать симптомы и отсрочить правильную постановку диагноза. Перечень всех особых указаний представлен в инструкции по медицинскому применению. Полная информация по препарату содержится в инструкции по медицинскому применениюо Дата выхода рекламного модуля: февраль, 2017 
Вероятно, влияние оказывали другие сопутствующие заболевания и стиль жизни. В популяционном канадском исследовании оценивалась минеральная плотность костей через 5 и 10 лет приема ИПП. Снижения минеральной плотности в динамике не обнаружено; интересно, что у больных, принимавших ИПП, уже изначально определялась более низкая плотность [26]. В 2010 г. FDA выпустило указание о необходимости предупреждающей надписи на этикетке ИПП о повышении риска переломов. Позже принято решение о безопасности безрецептурного применение ИПП сроком до 2 нед. до 3 циклов в год.

\section{Дексилант ${ }^{\circledR}$ зарегистрирован для лечения ГЭРБ (как НЭРБ, так и эрозивного эзофагита), а также для профилактики обострений эрозивного эзофагита}

Большой интерес вызывает потенциальная способность ИПП повышать плотность костей - за счет подавления активности остеокластов, в вакуолях которых экспрессирована протонная помпа [27]. При наличии показаний и соблюдении дозы оснований избегать назначения ИПП в группах риска остеопороза нет и нет необходимости дополнительного назначения препаратов кальция.

В мире зарегистрировано несколько десятков случаев выраженной гипомагниемии на фоне приема ИПП (при отсутствии признаков потери через кишечник и с мочой). Теоретически этот эффект можно связать с угнетением функции переносчиков магния в тонкой кишке; наклонность, по-видимому, определяется генетическими факторами. Этот эффект может развиться на любом сроке приема ИПП и регрессирует после отмены. FDA выпустило соответствующее предупреждение в отношении применения всего класса ИПП, хотя при описании случаев первоначально упоминались омепразол и эзомепразол [21]. Контроль магниемии перед началом терапии недостаточно обоснован, особенно при проведении коротких курсов. Более внимательный контроль нужен в случаях наличия мальабсорбции или заболевания почек; в этих ситуациях ИПП не противопоказаны [21].

В отношении риска развития дефицита «пищевого» витамина $B_{12}$ при длительном применении ИПП данные также противоречивые [28]. Всасывание витамина $\mathrm{B}_{12}$ в составе лекарственных средств и добавок не нарушается.

Выше уже упоминалось возможное взаимодействие ИПП и клопидогрела. In vitro, в которых показано фармакодинамическое взаимодействие, наблюдалось ослабление антитромботического эффекта по данным аденозин-дифосфат-индуцированной агрегации и повышенная активность тромбоцитов. Этот эффект, по всей вероятности, связан с пониженной функцией аллелей CYP2C19*2 и 3 (от 30 до 55\% в зависимости от расовой и национальной принадлежности) [21]. Эти полиморфизмы достаточно широко распространены, поэтому такое конкурентное взаимодействие ИПП и клопидо- грела необходимо учитывать. FDA рекомендовало не применять клопидогрел совместно с мощными ингибиторами СYР2С19: омепразолом, эзомепразолом и циметидином, хотя метаанализ не показал различий риска при применении ИПП различных классов вместе с клопидогрелом [29], а при анализе базы данных не показано достоверной связи между крупными сердечно-сосудистыми событиями и приемом клопидогрела при постоянном приеме, «переключении» и отмене ИПП [30]. Таким образом, с учетом отсутствия однозначных оценок риска при одновременном приеме ИПП и клопидогрела основное значение имеет надлежащая оценка факторов риска у пациента.

При применении ИПП вместе с высокими дозами метотрексата вероятно развитие токсических явлений последнего, что связано с замедлением его метаболизма [21].

В некоторых работах показана ассоциация приема ИПП с развитием внегоспитальной пневмонии [21]. В метаанализе (с анализом историй 120863 больных) не обнаружено связи пневмонии с применением ИПП дольше 180 дней (ОШ = 1,10; 95\% ДИ = 1,00-1,21); связь была более отчетливой для применения ИПП менее 30 дней (ОШ $=1,65 ; 95 \%$ ДИ = 1,25-2,19) и высоких доз ИПП (ОШ = 1,50; $95 \%$ ДИ = 1,33-1,68). Однако другие исследования и метаанализ связи не показали [21].

В отношении повышенного риска развития инфекции Clostridium difficile на фоне приема ИПП данные также неоднозначные [21]. Теоретически подавление продукции соляной кислоты может способствовать выживанию спор и их переходу в вегетативные формы. Различные метаанализы дают противоположные результаты, что может быть связано с недоучетом дополнительных факторов. Таким образом, сегодня нельзя считать доказанным повышенный риск развития этой инфекции при приеме ИПП, однако необходимо принимать во внимание данные о повышенной частоте ее развития среди больных, принимающих ИПП, и назначать эти препараты только с учетом показаний [21].

\section{Больщой интерес вызывает потенциальная способность ИПП повышать плотность костей - за счет подавления активности остеокластов, в вакуолях которых экспрессирована протонная помпа}

Аналогичные выводы можно сделать в отношении риска развития диареи путешественников. Международное общество медицины путешествий не рассматривает целесообразным отмену ИПП при поездке в районы высокого риска кишечных инфекций, хотя отмена может быть оправданной на короткий период при наличии индивидуального риска у больного [21]. Связь продолжительного приема ИПП (особенно более 1 года и у лиц старше 50 лет) с развитием избыточного бактериального роста в кишечнике показана, однако клиническое значение этого остается неясным [21] 
Более отчетливая связь применения ИПП прослеживается со спонтанным бактериальным перитонитом у пациентов с циррозом печени, находящихся в условиях стационара (ОШ = 4,3; 95\% ДИ = 1,3-11,7) [31], хотя есть и противоречивые данные [21]. В связи с этим необходимо тщательно взвешивать показания к назначению ИПП при циррозе печени с асцитом (особенно при низком содержании белка в асцитической жидкости) [21].

Описаны случаи развития острого интерстициального нефрита спустя достаточно короткий отрезок времени после начала приема ИПП [21].

Таким образом, хотя связь многих из перечисленных состояний с приемом ИПП полностью не доказана, при назначении препаратов этого класса необходимо соблюдать определенную осторожность. Анализ врачебных назначений в Дании показал частую необоснованность ИПП и отсутствие соответствующих показаний в медицинской документации [32]. ИПП должны назначаться только при наличии показаний, в рекомендованных дозах и на протяжении отрезка времени, рекомендованного для конкретного заболевания. В режиме безрецептурного отпуска, т. е. приема в течение не более 2 нед., для купирования изжоги, возникающей два и более раза в неделю, ИПП, в частности декслансопразол, рассматриваются как самый безопасный класс препаратов.

\section{ЛИТЕРАТУРА}

1. Ивашкин В.Т., Шептулин А.А., Трухманов А.С., Маев И.В. и др. Гастроэзофагеальная рефлюксная болезнь. Клинические рекомендации по диагностике и лечению. М., 2014. 31 с

2. Camilleri M, Malhi H, Acosta A. Gastrointestinal Complications of Obesity. Gastroenterology, 2017 May, 152(7): 1656-1670. doi: 10.1053/j.gastro.2016.12.052.

3. Vaishnav B, Bamanikar A, Maske P, Reddy A, Dasgupta S. Gastroesophageal Reflux Disease and its Association with Body Mass Index: Clinical and Endoscopic Study. J Clin Diagn Res, 2017 Apr, 11(4): OC01-OC04. doi: 10.7860/JCDR/2017/24151.9562.

4. Lee SW, Lee TY, Lien HC, Peng YC, Yeh HJ, Chang CS. Correlation Between Symptom Severity and Health-Related Life Quality of a Population With Gastroesophageal Reflux Disease. Gastroenterology Res, 2017 Apr, 10(2): 78-83. doi: 10.14740/gr753w.

5. Ida T, Inamori M, Inoh Y, Fujita K, Hamanaka J, Chiba H, Kusakabe A, Morohashi T, Goto T, Maeda S.Clinical Characteristics of Severe Er osive Esophagitis among Patients with Erosive Esophagitis: A Case-control Study. Intern Med, 2017, 56(11): 1293-1300. doi: 10.2169/internalmedicine.56.8058.

6. Filiberti RA, Fontana V, De Ceglie A, Blanchi S, Grossi E, Della Casa D, Lacchin T, De Matthaeis M, Ignomirelli O, Cappiello R, Rosa A, Foti M, Laterza F, D'Onofrio V, laquinto $\mathrm{G}$, Conio M. Alcohol consumption pattern and risk of Barrett's oesophagus and erosive oesophagitis: an Italian case-control study. Br J Nutr, 2017 May 8: 1-11. doi: 10.1017/ S0007114517000940.

7. Katz PO, Gerson LB, Vela MF. Guidelines for the diagnosis and management of gastroesophageal reflux disease. Am J Gastroenterol, 2013, 108: 308.

8. Li XQ, Andersson TB, Ahlström M, Weidolf LComparison of inhibitory effects of the proton pump-inhibiting drugs omeprazole, esomeprazole, lansoprazole, pantoprazole, and rabeprazole on human cytochrome P450 activities. Drug Metab Dispos, 2004 Aug, 32(8): 821-7.

9. Wedemeyer RS, Blume H. Pharmacokinetic drug interaction profiles of proton pump inhibitor: an update. Drug Saf, $2014 \mathrm{Apr}$, 37(4): 201-11. doi: 10.1007/s40264-014-0144-0.

10. BMS. Plavix prescribing information. 2011. http://products.sanofi.us/plavix/plavix html. Last accessed 23 April 2012.

11. Andersson T, Weidolf L. Stereoselective disposition of proton pump inhibitors. Clin Drug Investig, 2008, 28(5): 263-79.

12. Mermelstein J, Mermelstein AC, Chait MM Proton pump inhibitors for the treatment of patients with erosive esophagitis and gastroesophageal reflux disease: current evidence and safety of dexlansoprazole. Clin Exp Gastroenterol, 2016, 9: 163-172.

13. Hershcovici T, Jha LK, Fass R. Dexlansoprazole MR - a review. Ann Med, 2011, 43(5): 366-374.

14. Kukulka M, Eisenberg C, Nudurupati S. Comparator $\mathrm{pH}$ study to evaluate the single-dose pharmacodynamics of dual delayed-release dexlansoprazole $60 \mathrm{mg}$ and delayed-release esomeprazole $40 \mathrm{mg}$. Clin Exp Gastroenterol, 2011, 4(4): 213-220.

15. Lee RD, Vakily M, Mulford D, Wu J, Atkinson SN. Clinical trial: the effect and timing of food on the pharmacokinetics and pharmacodynamics of dexlansoprazole MR, a novel dual delayed release formulation of a proton pump inhibitor - evidence for dosing flexibility. Aliment Pharmacol Ther, 2009, 29(8) 824-833.

16. Lee RD, Mulford D, Wu J, Atkinson SN. The effect of time-of-day dosing on the pharmacokinetics and pharmacodynamics of dexlansoprazole MR: evidence for dosing flexibility with a Dual Delayed Release proton pump inhibitor. Aliment Pharmacol Ther, 2010, 31(9): 1001-1011.

17. Sharma P, Shaheen NJ, Perez MC, et al. Clinical trials: healing of erosive oesophagitis with dexlansoprazole MR, a proton pump inhibitor with a novel dual delayed-release formulation - results from two randomized controlled studies. Aliment Pharmacol Ther, 2009, 29(7): 731-741.

18. Peura DA, Pilmer B, Hunt B, Mody R, Perez MC The effects of increasing body mass index on heartburn severity, frequency and response to treatment with dexlansoprazole or lansoprazole. Aliment Pharmacol Ther, 2013, 37(8) 810-818.

19. Metz DC, Howden CW, Perez MC, Larsen L, O’Neil J, Atkinson SN. Clinical trial: dexlansoprazole MR, a proton pump inhibitor with dual delayed-release technology, effectively controls symptoms and prevents relapse in patients with healed erosive oesophagitis. Aliment Pharmacol Ther, 2009, 29(7): 742-754.

20. Iwakiri K, Sakurai Y, Shiino M, Okamoto $\mathrm{H}$, Kudou K, Nishimura A, Hiramatsu $\mathrm{N}$, Umegaki E, Ashida K. A randomized, doubleblind study to evaluate the acid-inhibitory effect of vonoprazan (20 mg and $40 \mathrm{mg}$ ) in patients with proton-pump inhibitor-resistant erosive esophagitis. Therap Adv Gastroenterol, 2017 Jun, 10(6): 439-451. doi: 10.1177/1756283X17705329.

21. Johnson DA, Oldfield EC. Reported Side Effects and Complications of Long-term Proton Pump
Inhibitor Use. Clin Gastroenterol Hepatol, 2013, 11(5): 458-464

22. McColl KE. Effect of proton pump inhibitors on vitamins and iron. Am J Gastroenterol, 2009. 104(Suppl 2): S5-S9.

23. Sarzynski E, Puttarajappa C, Xie Y, et al. Association between proton pump inhibitor use and anemia: a retrospective cohort study. Dig Dis Sci, 2011, 56: 2243-2253

24. Wright MJ, Sullivan RR, Gaffney-Stomberg E, et al. Inhibiting gastric acid production does not affect intestinal calcium absorption in young, healthy individuals: a randomized, crossover, controlled clinical trial. J Bone Miner Res, 2010, 25: 2205-2211.

25. Hansen KE, Jones AN, Lindstrom JM, et al. Do proton pump inhibitors decrease calcium absorption? J Bone Miner Res, 2010, 25: 25102519.

26. Targownik LE, Leslie WD, Davison KS, et al. The relationship between proton pump inhibitor use and longitudinal change in bone mineral density: a population-based study from the Canadian Multicentre Osteoporosis Study (CaMos). Am J Gastroenterol, 2012, 107: 1361-1369.

27. Mizunashi K, Furukawa Y, Katano K, et al. Effect of omeprazole, an inhibitor of $\mathrm{H}+, \mathrm{K}(+)$-ATPase, on bone resorption in humans. Calcif Tissue Int, 1993, 53: $21-25$.

28. Heidelbaugh J, Kim AH, Chang R, et al. Overutilization of protonpump inhibitors: what the clinician needs to know. Therap Adv Gastroenterol, 2012, 5: 219-232.

29. Kwok CS, Jeevanantham V, Dawn B, Loke YK. No consistent evidence of differential cardiovascular risk amongst proton-pump inhibitors when used with clopidogrel: meta-analysis. Int $\mathrm{J}$ Cardiol, 2013 Aug 10, 167(3): 965-74. doi: 10.1016/j.ijcard.2012.03.085

30. Banerjee S, Weideman RA, Weideman MW, et al. Effect of concomitant use of clopidogrel and proton pump inhibitors after percutaneous coronary intervention. Am J Cardiol, 2011, 107: 871-878.

31. Bajaj JS, Zadvornova Y, Heuman DM, et al. Association of proton pump inhibitor therapy with spontaneous bacterial peritonitis in cirrhotic patients with ascites. Am J Gastroenterol, 2009, 104: 1130-1134

32. Haastrup PF, Paulsen MS, Christensen RD, Søndergaard J, Hansen JM, Jarbøl DE. Medical and non-medical predictors of initiating longterm use of proton pump inhibitors: a nationwide cohort study of first-time users during a 10-year period. Aliment Pharmacol Ther, 2016 Jul, 44(1): 78-87.

33. Инструкция по применению лекарственного препарата для медицинского применения Дексилант ${ }^{\circledR}$. Рег.уд. ЛП 002477 от 26.05.14. 